\title{
Incorporación de los derivados grasos de anilina en los quilomicrones linfáticos
}

\author{
Por V. Ruiz-Gutiérrez y R. Maestro Durán \\ Instituto de la Grasa y sus Derivados (CSIC) \\ Aptdo. 1078. 41012-Sevilla
}

\section{RESUMEN}

Incorporación de los derivados grasos de anilina en los quilomicrones linfáticos.

Se han obtenido por sintesis química algunas sustancias que habian sido aisiadas, además de las anilidas grasas de aceites relacionados con el "síndrome tóxico". Estas sustancias son el 3-fenilamino-1,2 propanodiol y sus mono- y diésteres de ácidos grasos. Estos productos se han administrado por via oral a ratas cuyo canal linfático habia sido previamente canulado. Se ha recogido la linfa a distintos intervalos de tiempo y se han aislado de ella los quilomicrones y su fracción lipídica. Se ha estudiado esta fracción por cromatografía en capa fina, comprobándose la presencia de estas sustancias, asi como un metabolito procedente de ellas. Estos datos sugieren que dichos productos se incorporan a la sangre por absorción linfática.

PALABRAS CLAVE: Aceite desnaturalizado - Anilina (derivados de) Linfa - Quilomicrones - 3-Fenilamino-1,2-propanodiol 3-Fenilamino-1,2-propanodiol (ésteres de).

\section{SUMMARY}

Incorporation of new aniline fatty derivatives in to linphatic chylomicrons

Some compounds, other than fatty anilides, which had been isolated from oil samples involved in the "toxic syndrome", has been obtained by chemical synthesis. These substances are 3-phenylamine-1,2 propanediol and its fatty acyl mono- and diesters derivatives. These products have been orally administered to rats whose lynphatic canal had previously been cannulated. Samples of lymph were taken periodically. After isolation of chylomicrons from the lynphatic samples and analysis of its lipids by thin layer chromatography, we were able to detect the presence of the above mentioned compounds as well as a metabolic product of them. These results suggest that these products are incorporated into the blood via lymphatic absortion.

KEY-WORDS: Aniline (derivatives of) - Chylomicrons - Denatured oil - Lymph - 2-Phenylamine-1,2-propanediol - 3-Phenylamine-1,2- propanediol (esters of).

\section{INTRODUCCION}

Está suficientemente demostrado que hay una estrecha relación estadística entre la intoxicación alimentaria conocida como "síndrome del aceite tóxico" y el consumo de aceite de colza desnaturalizado con anilina que, importado de Francia, se refinó y mezcló con aceites comestibles de muy baja calidad. la mayor parte de estas mezclas de aceites contenían de 500 a 2000 partes por millón de anilidas de ácidos grasos, principalmente oleoanilida. La posible toxicidad de estas anilidas ha sido estudiada en varios laboratorios, usando conejos, ratones, embriones de pollo, fibroblastos humanos y otros tipos de cultivos celulares, sin que haya sido posible demostrar taxativamente que las anilidas fueran la causa de la enfermedad (1). Por esta razón, la organización Mundial de la Salud recomendó que se investigase en los aceites la presencia de estos componentes anómalos.

En muchos de estos aceites se han detectado, además de las anilidas, otros componentes que tenían en su molécula grupos $-\mathrm{NH}_{2}$ o- $\mathrm{NH}-(2)$. Ha sido posible aislar e identificar los tres derivados de la anilina más importantes, que son el 3-fenilamino-1,2-propanodiol (FAP) y sus correspondientes mono- y diésteres con ácidos grasos (3) (4).

Las estructuras se establecieron por datos químicos y espectrofotométricos $y$, posteriormente, por comparación con muestras obtenidas por síntesis química (5). Se prepararon cantidades suficientes para hacer algunas pruebas toxicológicas.

Administrando a ratones por vía oral o intraperitoneal, se vio que la más tóxica de las tres sustancias era el $\mathrm{FAP}$, con una $\mathrm{DL}_{50}$ de $517 \mathrm{mg} / \mathrm{kg}$, siguiéndola en toxicidad el monoéster.

El diéster pareció inocuo, al menos durante los 21 días que duró la experiencia, aunque los animales, cuando fueron sacrificados, presentaban anomalías pulmonares (6).

Nos pareció interesante estudiar la naturaleza de la absorción y metabolismo de estas sustancias por vía linfática, ya que ésta es la vía normal del metabolismo de las grasas. En efecto, una vez realizada la digestión, los productos de hidrólisis de las grasas y de las sustancias asociadas a ellas atraviesan la pared intestinal y, después de una serie de transformaciones en el seno de la mucosa y submucosa intestinal, pasan a la linfa y de ésta a la sangre, que los distribuye a los distintos órganos de utilización o reserva. Era de esperar que los compuestos anómalos que habíamos encontrado en los aceites relacionados con el Síndrome Tóxico, dada su naturaleza química, tuvieran un comportamiento similar al de los lípidos. 
Para ello se administraron estas sustancias por vía oral a ratas cuyo canal linfático había sido previamente canulado. En la linfa, recogida a distintos intervalos de tiempo, se aisló la fracción lipídica. Un estudio cromatográfico de ésta en capa fina de gel de sílice permitió comprobar la presencia de dichas sustancias, así como la de un metabolito, procedente de ellas y que también contenía en su molécula un grupo de amina primaria o secundaria.

\section{PARTE EXPERIMENTAL}

\subsection{Sustancias anómalas en los aceites.}

Para la detección e identificación de los derivados de anilina se utilizaron más de sesenta aceites tóxicos proporcionados por la Refinería ITH y de Sevilla, por la Di- rección General de la Salud Pública y por autoridades de Madrid y Frómista (Palencia). También se empleó aceite de colza normal, comestible suministrado por la firma KOIPE.

Las sustancias anómalas se aislaron y purificaron por cromatografía en capa fina como se ha descrito en un trabajo anterior (5), y se han sintetizado como se ha descrito (6). La identidad entre los compuestos aislados de los aceites tóxicos y los sintetizados en el laboratorio se ha confirmado por reacciones coloreadas (7), cromatografía en capa fina usando varios disolventes (Tabla I), espectroscopias de ultravioleta, infrarroja y de masas, determinación del contenido en nitrógeno, hidrólisis ácida, alcalina y enzimática con lipasa pancreática (8) y análisis de ácidos grasos (9). Los puntos de fusión también coinciden con los descritos en la literatura (10), (11) y (12).

TABLA I

Cromatografía en capa fina sobre sílicagel de los derivados de la anilina

\begin{tabular}{|c|c|c|c|c|c|c|}
\hline \multirow[t]{2}{*}{ Valores de Rf } & \multicolumn{2}{|c|}{ FAP } & \multicolumn{2}{|c|}{ FAP-monoéster } & \multicolumn{2}{|c|}{ FAP-diéster } \\
\hline & sintético & aislado & sintético & aislado & sintético & aislado \\
\hline Acetato de etilo & 0,51 & 0,52 & 0,92 & 0,92 & 0,96 & 0,97 \\
\hline Diclorometano & 0,00 & 0,00 & 0,22 & 0,22 & 0,66 & 0,65 \\
\hline Benceno & 0,00 & 0,00 & 0,03 & 0,03 & 0,30 & 0,30 \\
\hline $\begin{array}{l}\text { Color con } \\
\text { p-DMACA }\end{array}$ & naranja & naranja & morado & morado & morado & morado \\
\hline
\end{tabular}

\subsection{Animales}

Se utilizaron 16 ratas Wistar, machos, de 300 gramos de peso, suministradas por Iffa Credo (Lion, Francia), alimentados con dieta $\mathrm{AO} 4$ de Panlab enriquecida en grasas $(10 \%$ de aceite refinado de girasol u oliva, $5 \%$ de fibras). Los animales tuvieron libre acceso al alimento y al agua; $y$ fueron divididos en los siguientes grupos experimentales: lab.

1. Grupo control. Alimentado con pienso A04 de Pan-

2. Grupo alimentado con aceite de oliva o girasol (5 animales con cada aceite) y al que se le suministró FAP.

3. Grupo alimentado con aceite de oliva o girasol (ídem al 2) y al que se le suministró FAP-monoéster.

4. Grupo alimentado como 2 y 3 al que se le suministró FAP-diéster.

Antes de la operación fueron anestesiados con pentobarbital sódico $(40 \mathrm{mg} / \mathrm{kg}$ ).

La canulación del conducto linfático se realizó con un catéter de polietileno de $0,87 \mathrm{~mm}$ de diámetro interior y $1,12 \mathrm{~mm}$ de diámetro exterior, a través de una incisión en la región abdominal a unos $5 \mathrm{~mm}$ por debajo del cartílago xifoides, siguiendo la técnica de Bollman (13).
La cánula se saca al exterior de la piel por el costado derecho del animal.

Una vez realizado el proceso quirúrgico, se mantuvo a los animales en jaulas de inmovilización tipo Bollman durante 72 horas. Durante este período se les suministró una solución de 0,5 gramos de cloruro sódico, 0,5 gramos de cloruro potásico y 50 gramos de glucosa por litro de agua.

\subsection{Administración de las sustancias tóxicas.}

Tanto el FAP como los mono- y diésteres se administraron a las ratas por vía oral. La dosis del diéster fue de 1 gramo y la de monoéster y FAP, de 0,5 gramos, emulsionadas las sustancias en 2 mililitros de aceite refinado de oliva o girasol.

\subsection{Recogida de la linfa.}

El flujo de linfa recogido en los distintos ensayos estuvo comprendido entre 0,10 y $0,17 \mathrm{ml} / \mathrm{hora}$. Las muestras de linfa se recogieron en un tubo humedecido con una solución acuosa de $\mathrm{Na}_{2}$ EDTA al $10 \%$ y mantenido en un baño de hielo. 
Se fue recogiendo la linfa a distintos intervalos de tiempo para estudiar en las distintas fracciones la presencia de las sustancias administradas y sus posibles metabolitos, así como la cinética del proceso.

\subsection{Separación de los quilomicrones.}

Las muestras de linfa se diluyeron con un volumen igual de solución de cloruro sódico al $0,9 \%$ y se mezclaron agitando enérgicamente. El aislamiento de los quilomicrones se realizó sometiendo las soluciones a una ultracentrifugación, durante 60 minutos, a $50.000 \mathrm{G}$ en una ultracentrífuga Beckman, con rotor $70 \mathrm{Ti}$.

Como medida relativa del índice de contenido en lípidos de cada muestra se usó el tanto por ciento en transmisión de 50 microlitros de linfa diluídos a $165 \mathrm{ml}$ con agua destilada y midiendo a $540 \mathrm{~nm}$ en un espectrofotómetro Kotran Spectrophotometer Model, de acuerdo con el método de Laher (14).

\subsection{Extracción de los lípidos.}

Los lípidos de los quilomicrones se extrajeron por el procedimiento de Folch (15), con mezclas cloroformo-metanol 2:1 (v/v).

El extracto se evaporó en rotavapor a temperatura ambiente.

El residuo se disolvió en cloroformo, se filtró y la parte soluble, concentrada a pequeño volumen se conservó en congelador a $-20^{\circ} \mathrm{C}$ hasta su análisis.

\subsection{Estudio cromatográfico de los extractos.}

Una vez extraídos los lípidos, se estudiaron por cromatografía en capa fina de gel de sílice $60 \mathrm{GF}_{254}$ de la casa Merck, de 0,25 mm de espesor. Como testigos se usaron soluciones de FAP y sus mono- y diésteres en cloroformo. Las placas se desarrollaron con diclorometano y con acetato de etilo, revelándose a continuación con p-DMACA.

\subsection{Análisis estadístico.}

Todos los datos de las tablas vienen expresados por la media \pm la desviación típica, siendo el nivel de significación $p<0.05$.

\section{RESULTADOS Y DISCUSION}

Todos los animales utilizados produjeron linfa opaca entre 1 y 7 horas después de la administración de las sustancias. La turbidez continuó aumentando entre las 7 y las 24 horas. A partir de este tiempo, la linfa recogida fue casi transparente, lo que indica que las mayores concentraciones de lípidos tienen lugar entre las 7 y 24 horas.

Los volúmenes de linfa obtenidos a los distintos tiempos, con sus intervalos de confianza, están reflejados en la Tabla II. En ella se observa que el flujo linfático del conducto torácico varía según qué derivado de la anilina va disuelto en los lípidos. Los valores oscilan entre 1,88 $\mathrm{ml} /$ hora para el monooleil-FAP y $2,29 \mathrm{ml} / \mathrm{h}$ para el dioleil-FAP.

\section{CANTIDADES DE LINFA}

\begin{tabular}{|c|c|c|c|c|c|c|c|}
\hline 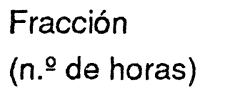 & $\begin{array}{c}1 \\
(0-4)\end{array}$ & $\begin{array}{c}2 \\
(4-8)\end{array}$ & $\begin{array}{c}3 \\
(8-12)\end{array}$ & $\begin{array}{c}4 \\
(12-24)\end{array}$ & $\begin{array}{c}5 \\
(24-30)\end{array}$ & $\begin{array}{c}\text { Total } \\
\mathrm{ml}\end{array}$ & $\mathrm{ml} / \mathrm{h}$ \\
\hline Control & $5^{\prime} 37 \pm 0 \prime 20$ & $8^{\prime} 35 \pm 0,31$ & $10^{\prime} 32 \pm 1 ' 36$ & $20^{\prime} 30 \pm 3^{\prime} 24$ & 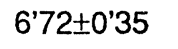 & 51,06 & 170 \\
\hline FAP & $5^{\prime} 62 \pm 0^{\prime} 30$ & $9^{\prime} 62 \pm 0^{\prime} 17$ & $10^{\prime} 32 \pm 1^{\prime} 45$ & $29^{\prime} 26 \pm 4^{\prime} 32^{* \star}$ & $6^{\prime} 62 \pm 0^{\prime} 43$ & $61^{\prime} 44$ & $2 ' 04$ \\
\hline FAP-monoéster & $6{ }^{\prime} 23 \pm 0^{\prime} 40$ & $7^{\prime} 48 \pm 00^{\prime} 22^{\prime \prime}$ & $17^{\prime} 24 \pm 3^{\prime} 27^{\star \star \star *}$ & $20 ’ 30 \pm 3^{\prime} 24$ & $5^{\prime} 23 \pm 1^{\prime} 21$ & $56^{\prime} 48$ & $1 ' 88$ \\
\hline FAP-diéster & $7^{\prime} 52 \pm 0^{\prime} 70^{*}$ & $8 ' 34 \pm 0,37$ & $16^{\prime} 20 \pm 2^{\prime} 40^{* *}$ & $28^{\prime} 32 \pm 4^{\prime} 40^{*}$ & $8^{\prime} 44 \pm 1^{\prime} 42^{*}$ & $68^{\prime} 82^{\star}$ & $29^{*}$ \\
\hline
\end{tabular}

Los resultados se expresan mediante la medida \pm S.D.

Diferencias significativas: " $p \leq 0$ 05, ${ }^{* *} p \leq 0,01,{ }^{* * *} p \leq 0,005$. 
El experimento modelo de este estudio nos ha suministrado una medida para evaluar la contribución del sistema linfático intestinal a la absorción de los nuevos derivados de la anilina encontrados en los aceites relacionados con el síndrome tóxico. Hasta ahora, todos los estudios realizados con estas sustancias (16) habían sido hechos sobre órganos, habiendo sido administrados a los animales por vía oral o intraperitoneal, utilizando aceite, agua o suero salino como vehículo. Siguiendo este tipo de administraciones observamos que en la sangre $y$ en los órganos se encontraron las sustancias tóxicas y algunos metabolitos, excretados fundamentalmente por el hígado vía biliar. Por ello ideamos este modelo de absorción para prevenir las complicaciones que nos daría el reciclaje enterohepático de los metabolitos biliares.

En este estudio se ha demostrado que la absorción de las sustancias anómalas no depende de la composición triglicerídica, ya que se han obtenido los mismos resultados usando como vehículo aceites distintos.
Por otra parte, es bien conocido que el sistema linfático gastrointestinal es la mayor y mejor ruta de absorción de ingredientes lipídicos (17) y de elementos traza liposolubles, como el DDT (1,1-dicloro-2,2 bis [4-etilfenilheptano]) (18) y el BP (benzo-a-pireno) (14).

El presente trabajo ha demostrado que, en el caso de las ratas, el transporte linfático juega un papel fundamental en la entrada sistemática de los tóxicos estudiados. Un seguimiento de los tóxicos en un período de 26 horas, combinando su detección en orina y heces, nos demuestra por métodos idénticos a los utilizados para la detección linfática que todas las sustancias tóxicas pasan a la linfa. Este tránsito de estas sustancias tóxicas es paralelo a la producción de quilomicrones por el enterocito, como lo refleja la turbidez y el contenido lipídico (Tabla II). Las pequeñas cantidades de linfa que se obtienen en las primeras fracciones (Tabla III) se deben, probablemente, al stress provocado en el animal por el período postoperatorio y la administración oral de las muestras.

TABLA III

Miligramos de lípidos en la linfa de 5 ratas por grupo intubadas con derivados de anilina

\section{CONTENIDO LIPIDICO EN LA LINFA}

\begin{tabular}{|c|c|c|c|c|c|c|c|}
\hline $\begin{array}{l}\text { Fracción } \\
\text { (n. horas) }\end{array}$ & $\begin{array}{c}1 \\
(0-4)\end{array}$ & $\begin{array}{c}2 \\
(4-8)\end{array}$ & $\begin{array}{c}3 \\
(8-12)\end{array}$ & $\begin{array}{c}4 \\
(12-24)\end{array}$ & $\begin{array}{c}5 \\
(24-30)\end{array}$ & $\begin{array}{l}\text { Total } \\
\mathrm{mg} / \mathrm{ml}\end{array}$ & $\begin{array}{c}\text { Total mg } \\
\text { recuperados }\end{array}$ \\
\hline Control & $8{ }^{\prime} 31 \pm 1^{\prime} 22$ & $16{ }^{\prime} 32 \pm 2 ' 2$ & $18{ }^{\prime} 31 \pm 1^{\prime} 2$ & $18{ }^{\prime} 34 \pm 1^{\prime} 3$ & $2^{\prime} 64 \pm 0^{\prime} 2$ & $63 ' 92$ & $958 ' 8$ \\
\hline FAP & $9^{\prime} 73 \pm 2 ' 4$ & $15^{\prime} 24 \pm 2{ }^{\prime} 3$ & $16^{\prime} 40 \pm 1^{\prime} 7$ & $21^{\prime} 56 \pm 2 ' 4$ & $3^{\prime} 70 \pm 0^{\prime} 1^{*}$ & $66 ' 63$ & $1.330^{\prime} 00^{* \star}$ \\
\hline FAP-monoéster & $3^{\prime} 23 \pm 1^{\prime} 4^{* \star \star *}$ & $17^{\prime} 32 \pm 1^{\prime} 2$ & $18{ }^{\prime} 20 \pm 1^{\prime} 3$ & $15^{\prime} 36 \pm 3^{\prime} 2^{*}$ & $2^{\prime} 36 \pm 0^{\prime} 2$ & $56^{\prime} 47$ & $787^{\prime} 65^{\star \star \star *}$ \\
\hline FAP-diéster & $10^{\prime} 43 \pm 1^{\prime} 2^{*}$ & $21^{\prime} 46 \pm 1^{\prime} 3^{*}$ & $19^{\prime} 23 \pm 4^{\prime} 8$ & $17 ' 24 \pm 177$ & $1^{\prime} 42 \pm 0^{\prime} 4^{* *}$ & $69^{\prime} 78^{\star \star *}$ & $1.067^{\prime} 62^{*}$ \\
\hline
\end{tabular}

Los resultados se expresan mediante la media \pm SD.

Diferencias singnificativas: * $p \leq 0^{\prime} 05,{ }^{* *} p \leq 0^{\prime} 01,{ }^{* * *} p \leq 0^{\prime} 005$.

La casi totalidad de los tóxicos se absorben en las primeras 24 horas después de la administración, lo que indica que prácticamente en este período desaparecen del lumen intestinal.

De los resultados obtenidos podemos deducir que las sustancias anómalas presentes en los aceites tóxicos, cuando se administran a las ratas emulsionadas en aceite (oliva o girasol), son absorbidas por las paredes intestinales. Los productos así absorbidos pueden ser transportados a los distintos órganos por vía linfática o sanguínea, pudiendo producir daños irreparables en los distintos lugares de asentamiento. Dichos daños fueron ya observados por nosotros en un trabajo anterior realizado con ratones (16), y que se traducían principalmente en esplenomegalias y enfisemas pulmonares. El que es- tos compuestos sean absorbidos vía linfática y conducidos por el conducto torácico a los pulmones podría explicar que el primer daño detectado en la epidemia fuera una neumonía atípica.

En el caso del diéster del FAP, hemos encontrado que en la fase clorofórmica de la fracción de lípidos aparece la sustancia administrada, parcialmente hidrolizada a monoéster y a base libre. En la fracción metanólica aparece una sustancia, cuya naturaleza desconocemos, que en cromatografía en capa fina sobre gel de silice tiene los siguientes valores de $\mathrm{Rf}$ : acetato de etilo-cloroformo $3: 1(\mathrm{v} / \mathrm{v})=0,40$; acetato de etilo: 0,76 ; diclorometano: 0,04 ; da color positivo (morado) con p-DMACA, lo que indica la presencia de un grupo $-\mathrm{NH}_{2}$ o - $\mathrm{NH}$ - en la molécula, y es un metabolito del diéster suministrado a los animales, puesto que no aparece en la 
linfa de aquéllos a los que se les había dado sólo aceite de girasol.

También hemos encontrado este metabolito en la fracción metanólica de los lípidos de la linfa de animales a los que se había administrado el monoéster. En la fracción clorofórmica encontramos el monoéster administrado, parcialmente hidrolizado a base libre.

El 3-fenilamino-1,2-propanodiol, por su carácter más polar, apareció repartido entre ambas fases, metanólica y clorofórmica. La mayor parte del compuesto se había transformado en el metabolito ya citado anteriomente, cuya naturaleza desconocemos pero sobre la cual seguimos investigando.

\section{BIBLIOGRAFIA}

1. Organización Mundial de la Salud.- El síndrome del aceite tóxico. Informe sobre la Reunión de Madrid del 21-25 de Marzo de 1983. Editado por el Ministerio de Sanidad y Consumo. Madrid, 1984.

2. Vázquez-Roncero, A., Maestro-Durán, R., Graciani-Constante, E. y Janer del Valle, C.- "Aceites vegetales desnaturalizados con anilina. 1. Análisis por cromatografia en capa fina".- Grasas y Aceites 33 (1982) 281-286.

3. Vázquez-Roncero, A. Janer del Valle, C. Maestro-Durán, R. y Graciani-Constante, E.- "New Aniline Derivatives in cooking Oils associated with the Toxic Oil Syndrome". - The Lancet 29 (1983) 1024.

4. Vázquez-Roncero, A., Maestro-Durán, R. y Ruiz-Gutiérrez, V.- "New Aniline Derivatives in Toxic Oil Syndrome. Toxicity in mice of 3-phenylamino-1,2-propanediol and its fatty acid mono-and diesters".Grasas y Aceites 35 (1984) 330-331.

5. Vázquez-Roncero, A., Maestro-Durán, R., Graciani-Constante, E. y Janer del Valle, C.- "Nuevos derivados de la anilina en aceites relacionados con el sindrome tóxico. I. Esteres grasos de 1,2-propanodiol-3-aminofenilo".- Grasas y Aceites 35 (1984) 15-21.

6. Vázquez-Roncero, A. Maestro-Durán, R Janer del Valle, $\mathrm{C}$. Graciani-Constante, E. y Gómez, R.- "Nuevos derivados de la anilina en aceites asociados al síndrome tóxico. II. Sintesis de mono- y diésteres del 3-fenilamino-1,2-propanodiol".- Grasas y Aceites 36 (1985) 382-389.
7. Sakai, S., Suzuki, K., Nori, H. y Fujuro, M.- "Color reaction of amines with p-dimethylaminocinnamaldehyde".- Japan Analyst 9 (1960) 862-864. Tomado de Z. Ana. Chem. 183 (1961) 450.

8. Norma UNE 55-079-73.- "Materias grasas. Determinación cualitativa y cuantitativa de los ácidos grasos situados en la posición beta de los triglicéridos".

9. Norma UNE 55-037-73.- "Materias grasas. Determinación de ácidos grasos por cromatografía gaseosa".

10. Malinovski, M.S. y Perchik, V.N.,- "Condensation of glycidol with aromatic amines".- Zhur. Obschei khim. 27 (1957) 1591-1593. Según Chem. Abst. 52 (1957) $3721 \mathrm{~cd}$.

11. Sminova, T.V. y Sapunov., V.N.- "Synthesis of some N-derivatives of 2,3-dihydroxy-propylamine". -Zh. vses. Khgim. obsch im D.I. Mendaleeva 8 (1963) 235-237. Según Chem. Abst. 59 (1963) 5.0501.

12. Fauran, C. y Douzon.- "Une nouvelle série d'antidepresseurs: les derivés de l'hydroxymétyl-5-oxazolidinona-2".- Chim. Thérap. (1973) 324-327.

13. Bamberger, E. y Kitschelt., M.- "Ueber eine synthese des Chinolins und Skatols", - Ber. Deuts. Chem. Ges. 27 (1984) 3.421-3.427.

14. Rouselle, D., Gilbert, J. y Rumpf, P.- "Lactones à cycle hepta-atomique condensé avec un noyau aromatique. II. Dérivés de la benzooxa-4-azépine-1-one-5".- Bull. Soc. Chim. France (1975) 283-285.

15. Bollman, J.J., Cain, V.C. y Grinday, J.H.- "Techniques for the collection of limph from the liver, small intestine or thoracic duck of the rat". $-J$. lab. and Chim. Med. 33 (1948) 1349.

16. Folch, J., Lees, M. y Stanley, G.H.S.- "A simple method for the isolation and prurfication of total lipids from animal tissues"- J. Biol. Chem. 226 (1957) 497-509.

17. Maestro-Durán, R., Ruiz-Gutiérrez, V. y Vázquez-Roncero, A.- "Estudio toxicológico de nuevos derivados de la anilina presentes en aceites tóxicos".- Revista de Toxicología 2 (1985) 168-183.

18. Barrowman, J.A.- "Physiology of the gastrointestinal lymphatic system".- Cambridge University Press. Cambridge, 1978.

19. Pocock, D.M.E. y Vost, A.- "DDT absrotion and chylomicrom transport in rat".- Lipids 9 (1974) 374-383.

20. Laher, J.M., Rigler, M.W., Vetter, R.D., Barrowman, J.A. y Patton, J.S.- "Similar bioavaility and lymphatic transport of benzo-(a) pyrene when administered to rats in different amounts of dietary fats".- $\mathrm{J}$ Lipid Res. 25 (1984) 1337-1342.

(Recibido: Mayo 1991) 